\title{
Mental Health and Substance Use Associated with Hospitalization among People with COVID-19: A Population-Based Cohort Study
}

\author{
Héctor Alexander Velásquez García 1,2, James Wilton ${ }^{1}$, Kate Smolina 1,2, Mei Chong 1, Drona Rasali 1,2 (D), \\ Michael Otterstatter ${ }^{1,2}$, Caren Rose ${ }^{1,2}$, Natalie Prystajecky ${ }^{1}$, Samara David ${ }^{1} \mathbb{D}$, Eleni Galanis ${ }^{1,2}$, \\ Geoffrey McKee ${ }^{1}$, Mel Krajden ${ }^{1,3}$ and Naveed Zafar Janjua 1,2,4,*
}

1 British Columbia Centre for Disease Control, Vancouver, BC V5Z 4R4, Canada; hector.velasquez@bccdc.ca (H.A.V.G.); james.wilton@bccdc.ca (J.W.); kate.smolina@bccdc.ca (K.S.); mei.chong@bccdc.ca (M.C.); drona.rasali@bccdc.ca (D.R.); michael.otterstatter@bccdc.ca (M.O.); caren.rose@bccdc.ca (C.R.); natalie.prystajecky@bccdc.ca (N.P.); samara.david@phac-aspc.gc.ca (S.D.); eleni.galanis@bccdc.ca (E.G.); geoffrey.mckee@bccdc.ca (G.M.); mel.krajden@bccdc.ca (M.K.)

2 School of Population and Public Health, University of British Columbia, Vancouver, BC V6T 1Z3, Canada

3 Department of Pathology and Laboratory Medicine, University of British Columbia, Vancouver, BC V6T 1Z7, Canada

check for updates

Citation: Velásquez García, H.A.; Wilton, J.; Smolina, K.; Chong, M.; Rasali, D.; Otterstatter, M.; Rose, C.; Prystajecky, N.; David, S.; Galanis, E.; et al. Mental Health and Substance Use Associated with Hospitalization among People with COVID-19: A Population-Based Cohort Study. Viruses 2021, 13, 2196. https:/ / doi.org/10.3390/v13112196

Academic Editors:

Dimitrios Paraskevis,

Maria Yavropoulou and

Sotirios Tsiodras

Received: 29 September 2021

Accepted: 28 October 2021

Published: 31 October 2021

Publisher's Note: MDPI stays neutral with regard to jurisdictional claims in published maps and institutional affiliations.

Copyright: (C) 2021 by the authors Licensee MDPI, Basel, Switzerland. This article is an open access article distributed under the terms and conditions of the Creative Commons Attribution (CC BY) license (https:/ / creativecommons.org/licenses/by/ $4.0 /)$.
4 Centre for Health Evaluation and Outcome Sciences, St. Paul's Hospital, Vancouver, BC V6Z 1Y6, Canada

* Correspondence: naveed.janjua@bccdc.ca; Tel.: +1-604-707-2514

\begin{abstract}
This study identified factors associated with hospital admission among people with laboratory-diagnosed COVID-19 cases in British Columbia. The study used data from the BC COVID-19 Cohort, which integrates data on all COVID-19 cases with data on hospitalizations, medical visits, emergency room visits, prescription drugs, chronic conditions and deaths. The analysis included all laboratory-diagnosed COVID-19 cases in British Columbia to 15 January 2021. We evaluated factors associated with hospital admission using multivariable Poisson regression analysis with robust error variance. Of the 56,874 COVID-19 cases included in the analysis, 2298 were hospitalized. Factors associated with increased hospitalization risk were as follows: male sex (adjusted risk ratio $(\mathrm{aRR})=1.27 ; 95 \% \mathrm{CI}=1.17-1.37)$, older age $(p$-trend $<0.0001$ across age groups increasing hospitalization risk with increasing age [aRR 30-39 years $=3.06 ; 95 \% \mathrm{CI}=2.32-4.03$, to aRR $80+$ years $=43.68 ; 95 \% \mathrm{CI}=33.41-57.10$ compared to $20-29$ years-old $])$, asthma $(\mathrm{aRR}=1.15$; $95 \% \mathrm{CI}=1.04-1.26)$, cancer $(\mathrm{aRR}=1.19 ; 95 \% \mathrm{CI}=1.09-1.29)$, chronic kidney disease $(\mathrm{aRR}=1.32$; $95 \% \mathrm{CI}=1.19-1.47$ ), diabetes (treated without insulin $\mathrm{aRR}=1.13 ; 95 \% \mathrm{CI}=1.03-1.25$, requiring insulin $\mathrm{aRR}=5.05 ; 95 \% \mathrm{CI}=4.43-5.76)$, hypertension $(\mathrm{aRR}=1.19 ; 95 \% \mathrm{CI}=1.08-1.31)$, injection drug use $(\mathrm{aRR}=2.51 ; 95 \% \mathrm{CI}=2.14-2.95)$, intellectual and developmental disabilities $(\mathrm{aRR}=1.67$; $95 \% \mathrm{CI}=1.05-2.66)$, problematic alcohol use $(\mathrm{aRR}=1.63$; 95\% CI $=1.43-1.85)$, immunosuppression $(\mathrm{aRR}=1.29 ; 95 \% \mathrm{CI}=1.09-1.53)$, and schizophrenia and psychotic disorders $(\mathrm{aRR}=1.49 ; 95 \%$ $\mathrm{CI}=1.23-1.82)$. In an analysis restricted to women of reproductive age, pregnancy $(\mathrm{aRR}=2.69$; $95 \% \mathrm{CI}=1.42-5.07)$ was associated with increased risk of hospital admission. Older age, male sex, substance use, intellectual and developmental disability, chronic comorbidities, and pregnancy increase the risk of COVID-19-related hospitalization.
\end{abstract}

Keywords: COVID-19; cohort studies; registries; risk factors; hospitalization; mental health; substancerelated disorders; diabetes mellitus; intellectual disability; pregnancy

\section{Introduction}

COVID-19 caused by SARS-CoV-2, has affected millions of people globally and can generate a spectrum of health outcomes among those infected. Clinical presentation can range from asymptomatic/mild illnesses to severe disease that requires hospitalization and intensive care [1-3]. 
The overall goal of the pandemic response is to minimize severe disease, overall deaths and societal disruption. Various demographic factors (e.g., older age, male sex) and chronic comorbidities (e.g., diabetes, cardiovascular disease (CVD), hypertension) have been identified as risk factors for hospitalization and other severe outcomes [2]. However, most studies have been conducted on patients presenting at hospitals, and there are few population-based studies evaluating risk factors in all COVID-19-diagnosed individuals in a specific jurisdiction. Limiting analyses to hospitalized patients may lead to potential selection bias when characterizing risk factors. Furthermore, even though older age has been identified as the strongest risk factor for severe disease along with various comorbidities [4], very few studies have investigated the relationship of substance use, intellectual disabilities and insulin-dependent diabetes with the risk of severe outcomes [5,6].

Identification of risk factors for COVID-19 hospitalization is important for the prioritization of interventions aimed at reducing health system burden and maintaining hospital capacity. However, risk factors may differ by jurisdiction as the evolution of the COVID-19 pandemic has not been uniform globally. Further, the clinical threshold for hospital admission may vary across settings, especially early in the pandemic. In this study, we identified factors associated with hospital admission among people with COVID-19 infection in British Columbia (BC).

\section{Materials and Methods}

\subsection{Study Population}

This study used data from the BC COVID-19 Cohort (BCC19C), which integrates data on all individuals tested for COVID-19 in BC, with data on COVID-19 hospital and ICU admissions, medical visits, other hospitalizations, emergency room visits, prescription drugs, chronic conditions and mortality (Supplementary Materials Table S1). The BCC19C was established as a public health surveillance system under the BCCDC's public health mandate. This study was reviewed and approved by the Behavioural Research Ethics Board at the University of British Columbia (approval \# H20-02097).

The study population for this analysis included individuals who tested positive for SARS-CoV-2 by real-time reverse transcription-polymerase chain reaction (RT-PCR), from 26 January 2020 to 15 January 2021. The outcome of interest was hospitalization (as a measure of COVID-19 severity), defined as hospital admission in a BC acute care facility within 14 days after a positive SARS-CoV-2 test [7-9]. Patients residing in long term care facilities were excluded from the analyses as their transfer to hospitals was variable over time and across local regions. For women of reproductive age (15-49 years), hospital admissions were only considered related to COVID-19 if no obstetric-related hospitalization codes were found in the Discharge Abstract Database (DAD) within 14 days of hospital admission in Appendix B of Supplementary Materials.

\subsection{Comorbidities}

We examined the following chronic conditions: Alzheimer/dementia, asthma, chronic heart disease (CHD: acute myocardial infarct, angina, heart failure, ischemic myocardial infarct), chronic obstructive pulmonary disease (COPD), cirrhosis, chronic kidney disease (CKD), depression, diabetes (categorized as no-diabetes, treated without insulin and requiring insulin), epilepsy, gout, hypertension, stroke (ischemic, haemorrhagic, transitory ischemic attack), mood and anxiety disorders, osteoarthritis, osteoporosis, parkinsonism, rheumatoid arthritis, substance use disorder, injection drug use (IDU), problematic alcohol use, cancer, immunosuppression, intellectual and developmental disabilities (IDD) and schizophrenia and psychotic disorders (SZP). Variable definitions and diagnostic codes used to identify comorbidities are detailed in Appendix C of Supplementary Materials.

\subsection{Statistical Analysis}

We described the baseline characteristics of participants including age, sex and pregnancy status. We evaluated risk factors associated with hospital admission, calculating 
risk ratios through multivariable Poisson regression models with robust error variance [10]. Analyses were conducted treating age as continuous as well as categorized into groups. Model-building started with a model including age and sex, followed by other variables. Added variables were evaluated through Wald's tests and improvement of model fit was determined through the Akaike Information Criterion. To assess population differences across time, the cohort was stratified according to two waves or time periods: 26 January to 1 August 2020 and 2 August 2020 to 15 January 2021. Sensitivity analyses were performed by (1) stratifying the population by age group and (2) by restricting the outcome to hospitalizations lasting more than two days to address severity. All statistical analyses were performed using R version 4.0.2 [11].

\section{Results}

\subsection{Demographic and Clinical Characteristics}

The analysis included 56,874 COVID-19 cases diagnosed before 15 January 2021, 2298 $(4.0 \%)$ of whom were hospitalized. Males represented $51.2 \%$ of the people reported as COVID-19 positive and a greater percentage of hospital admissions (58.5\%) (Table 1). The median age of COVID-19 cases was 35 years (IQR: 24-50), while that of people requiring hospital admission was nearly twice that (66 years; IQR: 53-78). The hospitalization rate increased gradually with each 10-year age group, from $0.2 \%$ in the youngest subpopulation ( $<20$ years) to $34.2 \%$ in the eldest group ( $80+$ years) $(R R=66.96 ; 95 \% C I=52.35-85.65)$. A higher proportion of pregnant women were hospitalized than women who were not pregnant $(2.5 \%$ vs. $1.1 \%)$.

\subsection{Chronic Comorbidities as Risk Factors}

The proportion of individuals with comorbidities among hospital admissions was higher than in those who did not require hospitalization (Table 1), including hypertension $(54.1 \%$ vs. $14 \%)$, depression ( $38.3 \%$ vs. $21.7 \%)$, diabetes ( $35.8 \%$ vs. $8.3 \%)$, osteoarthritis ( $27.3 \%$ vs. $6.6 \%)$, CHD ( $26.9 \%$ vs. $4.4 \%)$, cancer $(26.8 \%$ vs. $9.9 \%)$, CKD ( $23.5 \%$ vs. $3.0 \%)$, asthma ( $20.1 \%$ vs. $13.1 \%)$, substance use disorder ( $13.7 \%$ vs. $4.3 \%)$, problematic alcohol use ( $13.6 \%$ vs. $4.1 \%)$, heart failure ( $11.7 \%$ vs. $1.1 \%)$, COPD (11.5\% vs. $1.5 \%)$, IDU ( $10 \%$ vs. $3.8 \%)$ and immunosuppression (6.0\% vs. $2.2 \%)$.

In the adjusted multivariable Poisson regression model (Table 2; Figure 1), age ( $p$-trend $<0.0001$ across age groups with increasing risk with older age [aRR 30-39 years $=3.06$; $95 \%$ $\mathrm{CI}=2.32-4.03$, to aRR $80+$ years $=43.68 ; 95 \% \mathrm{CI}=33.41-57.10$ compared to $20-29$ years-old $]$ ), male sex $(\mathrm{aRR}=1.27 ; 95 \% \mathrm{CI}=1.17-1.37)$, asthma $(\mathrm{aRR}=1.15 ; 95 \% \mathrm{CI}=1.04-1.26)$, cancer $(1.19 ; 95 \% \mathrm{CI}=1.09-1.29), \mathrm{CKD}(\mathrm{RR}=1.32 ; 95 \% \mathrm{CI}=1.19-1.47)$, diabetes (treated without insulin aRR $=1.13 ; 95 \% \mathrm{CI}=1.03-1.25$, requiring insulin $\mathrm{aRR}=5.05 ; 95 \% \mathrm{CI}=4.43-5.76)$, hypertension $(\mathrm{aRR}=1.19 ; 95 \% \mathrm{CI}=1.08-1.31)$, immunosuppression $(1.30 ; 95 \% \mathrm{CI}=1.10-1.54)$, IDU $(\mathrm{aRR}=2.51 ; 95 \% \mathrm{CI}=2.14-2.95), \mathrm{IDD}(\mathrm{aRR}=1.67 ; 95 \% \mathrm{CI}=1.05-2.66)$, problematic alcohol use $(\mathrm{aRR}=1.63 ; 95 \% \mathrm{CI}=1.43-1.85)$ and $\mathrm{SZP}(\mathrm{aRR}=1.49 ; 95 \% \mathrm{CI}=1.23-1.82)$ were associated with increased hospitalization risk. The analysis by time period (Supplementary Tables S2 and S3) did not show any remarkable differences in risk factors. 
Table 1. Distribution of characteristics in the BC COVID-19 cohort (confirmed cases, $n=56,874$ ), according to hospitalization status.

\begin{tabular}{|c|c|c|c|c|c|c|c|}
\hline Variable & Category & $\begin{array}{c}\text { Total * } \\
n=56,874\end{array}$ & $\begin{array}{c}\text { Non-Hospitalized }^{\circ} \\
n=54,576\end{array}$ & $\begin{array}{c}\text { Hospitalized }^{\circ} \\
n=2298\end{array}$ & $\begin{array}{l}\text { Hospitalized } \\
\text { Row } \%\end{array}$ & $\begin{array}{c}\text { Crude Incidence Rate Ratio } \\
(95 \% \text { Conf. Interv. })\end{array}$ & $p^{\dagger}$ \\
\hline \multirow{2}{*}{ Sex } & Female & $27,769(48.8 \%)$ & $26,816(49.1 \%)$ & $953(41.5 \%)$ & $3.4 \%$ & Reference & \multirow{2}{*}{$<0.0001$} \\
\hline & Male & $29,105(51.2 \%)$ & $27,760(50.9 \%)$ & $1345(58.5 \%)$ & $4.6 \%$ & $1.35(1.24-1.46)$ & \\
\hline Age (years) & $\mathrm{N} / \mathrm{A}$ & $36(28)^{i}$ & $35(26)^{i}$ & $66(25)^{i}$ & - & $1.07(1.06-1.07)$ & $<0.0001$ \\
\hline \multirow{8}{*}{ Age group $\ddagger$} & $<20$ years & $7683(13.5 \%)$ & $7665(14.0 \%)$ & $18(0.8 \%)$ & $0.2 \%$ & $0.45(0.27-0.77)$ & 0.0031 \\
\hline & 20-29 years & $13,490(23.7 \%)$ & $13,421(24.6 \%)$ & $69(3.0 \%)$ & $0.5 \%$ & Reference & - \\
\hline & 30-39 years & $10,681(18.8 \%)$ & $10,507(19.3 \%)$ & $174(7.6 \%)$ & $1.6 \%$ & $3.18(2.41-4.20)$ & $<0.0001$ \\
\hline & $40-49$ years & $8818(15.8 \%)$ & $8604(15.8 \%)$ & $214(9.3 \%)$ & $2.4 \%$ & $4.74(3.62-6.22)$ & $<0.0001$ \\
\hline & $50-59$ years & $7565(13.3 \%)$ & $7201(13.2 \%)$ & $364(15.8 \%)$ & $4.8 \%$ & $9.41(7.28-12.15)$ & $<0.0001$ \\
\hline & 60-69 years & $4793(8.4 \%)$ & $4330(7.9 \%)$ & $463(20.1 \%)$ & $9.7 \%$ & $18.89(14.70-24.27)$ & $<0.0001$ \\
\hline & 70-79 years & $2425(4.3 \%)$ & $1915(3.5 \%)$ & $510(22.2 \%)$ & $21.0 \%$ & $41.12(32.10-52.67)$ & $<0.0001$ \\
\hline & $80+$ years & $1419(2.5 \%)$ & $933(1.7 \%)$ & $486(21.1 \%)$ & $34.2 \%$ & $66.96(52.35-85.65)$ & $<0.0001$ \\
\hline \multirow{2}{*}{$\begin{array}{c}\text { Pregnant } \\
\text { (female population, ages } \\
15 \text { to } 49 ; n=17,693 \text { ) }\end{array}$} & No & $16,638(94.0 \%)$ & $16,457(94.1 \%)$ & $181(86.6 \%)$ & $1.1 \%$ & Reference & - \\
\hline & Unknown & $657(3.7 \%)$ & $639(3.7 \%)$ & $18(8.6 \%)$ & $2.7 \%$ & $2.52(1.56-4.06)$ & 0.0002 \\
\hline \multirow{2}{*}{ Angina } & No & $55,915(98.3 \%)$ & $53,850(98.7 \%)$ & $2065(89.9 \%)$ & $3.7 \%$ & Reference & \multirow{2}{*}{$<0.0001$} \\
\hline & Yes & $959(1.7 \%)$ & $726(1.3 \%)$ & $233(10.1 \%)$ & $24.3 \%$ & $6.58(5.84-7.41)$ & \\
\hline \multirow{2}{*}{ Chronic heart disease $^{1}$} & No & $53,840(94.7 \%)$ & $52,161(95.6 \%)$ & $1679(73.1 \%)$ & $3.1 \%$ & Reference & \multirow{2}{*}{$<0.0001$} \\
\hline & Yes & $3034(5.3 \%)$ & $2415(4.4 \%)$ & $619(26.9 \%)$ & $20.4 \%$ & $6.54(6.01-7.12)$ & \\
\hline \multirow{2}{*}{ Heart failure } & No & $56,003(98.5 \%)$ & $53,975(98.9 \%)$ & $2028(88.3 \%)$ & $3.6 \%$ & Reference & \multirow{2}{*}{$<0.0001$} \\
\hline & Yes & $871(1.5 \%)$ & $601(1.1 \%)$ & $270(11.7 \%)$ & $31.0 \%$ & $8.56(7.68-8.54)$ & \\
\hline \multirow{2}{*}{ Hypertension } & No & $48,009(84.4 \%)$ & $46,955(86.0 \%)$ & $1054(45.9 \%)$ & $2.2 \%$ & Reference & \multirow{2}{*}{$<0.0001$} \\
\hline & Yes & $8865(15.6 \%)$ & $7621(14.0 \%)$ & $1244(54.1 \%)$ & $14.0 \%$ & $6.39(5.91-6.92)$ & \\
\hline
\end{tabular}


Table 1. Cont

\begin{tabular}{|c|c|c|c|c|c|c|c|}
\hline Variable & Category & $\begin{array}{c}\text { Total * } \\
n=56,874\end{array}$ & $\begin{array}{c}\text { Non-Hospitalized }^{\circ} \\
n=54,576\end{array}$ & $\begin{array}{l}\text { Hospitalized }^{\circ} \\
n=2298\end{array}$ & $\begin{array}{l}\text { Hospitalized } \\
\text { Row } \%\end{array}$ & $\begin{array}{l}\text { Crude Incidence Rate Ratio } \\
(95 \% \text { Conf. Interv. })\end{array}$ & $p^{+}$ \\
\hline \multirow{2}{*}{ Ischemic heart disease } & No & $54,139(95.2 \%)$ & $52,378(96.0 \%)$ & $1761(76.6 \%)$ & $3.3 \%$ & Reference & \multirow{2}{*}{$<0.0001$} \\
\hline & Yes & $2735(4.8 \%)$ & $2198(4.0 \%)$ & $537(23.4 \%)$ & $19.6 \%$ & $6.04(5.52-6.59)$ & \\
\hline \multirow{2}{*}{ Myocardial infarct } & No & $56,273(98.9 \%)$ & $54,109(99.1 \%)$ & $2164(94.2 \%)$ & $3.8 \%$ & Reference & \multirow{2}{*}{$<0.0001$} \\
\hline & Yes & $601(1.1 \%)$ & $467(0.9 \%)$ & $134(5.8 \%)$ & $22.3 \%$ & $5.80(4.97-6.77)$ & \\
\hline Immunosuppression $^{2}$ & No & $55,541(97.7 \%)$ & $53,380(97.8 \%)$ & $2161(94.0 \%)$ & $3.9 \%$ & Reference & $<0.0001$ \\
\hline \multirow{2}{*}{ Rheumatoid arthritis } & No & $56,198(98.8 \%)$ & $53,998(98.9 \%)$ & $2200(95.7 \%)$ & $3.9 \%$ & Reference & \multirow{2}{*}{$<0.0001$} \\
\hline & Yes & $676(1.2 \%)$ & $578(1.1 \%)$ & $98(4.3 \%)$ & $14.5 \%$ & $3.70(3.07-4.47)$ & \\
\hline \multirow{2}{*}{ Depression } & No & $44,541(78.3 \%)$ & $43,124(79.0 \%)$ & $1417(61.7 \%)$ & $3.2 \%$ & Reference & \multirow{2}{*}{$<0.0001$} \\
\hline & Yes & $12,333(21.7 \%)$ & $11,452(21.0 \%)$ & $881(38.3 \%)$ & $7.1 \%$ & $2.25(2.07-2.44)$ & \\
\hline \multirow{2}{*}{$\begin{array}{c}\text { Intellectual and } \\
\text { developmental disability }\end{array}$} & No & $56,444(99.2 \%)$ & $54,177(99.3 \%)$ & $2267(98.7 \%)$ & $4.0 \%$ & Reference & \multirow{2}{*}{0.0008} \\
\hline & Yes & $430(0.8 \%)$ & $399(0.7 \%)$ & $31(1.3 \%)$ & $7.2 \%$ & $1.79(1.28-2.53)$ & \\
\hline $\begin{array}{l}\text { Mood and anxiety } \\
\text { disorders }\end{array}$ & Yes & $15,284(26.9 \%)$ & $14,254(26.1 \%)$ & $1030(44.8 \%)$ & $6.7 \%$ & $2.21(2.04-2.39)$ & $<0.0001$ \\
\hline \multirow{2}{*}{$\begin{array}{l}\text { Schizophrenia and } \\
\text { psychotic disorders }\end{array}$} & No & $56,112(98.7 \%)$ & $53,927(98.8 \%)$ & $2185(95.1 \%)$ & $3.9 \%$ & Reference & \multirow{2}{*}{$<0.0001$} \\
\hline & Yes & $762(1.3 \%)$ & $649(1.2 \%)$ & $113(4.9 \%)$ & $14.8 \%$ & $3.81(3.20-4.53)$ & \\
\hline \multirow{3}{*}{ Diabetes } & No & $51,487(90.5 \%)$ & $50,011(91.6 \%)$ & $1476(64.2 \%)$ & $2.9 \%$ & Reference & - \\
\hline & Non-insulin & $5141(9.1 \%)$ & $4541(8.3 \%)$ & $600(26.1 \%)$ & $11.7 \%$ & $4.07(3.72-4.46)$ & $<0.0001$ \\
\hline & Insulin ${ }^{5}$ & $246(0.4 \%)$ & $24(0.0 \%)$ & $222(9.7)$ & $90.2 \%$ & $31.48(29.50-33.59)$ & $<0.0001$ \\
\hline \multirow{2}{*}{ Gout } & No & $55,707(97.9 \%)$ & $53,622(98.3 \%)$ & $2085(90.7 \%)$ & $3.7 \%$ & Reference & \multirow{2}{*}{$<0.0001$} \\
\hline & Yes & $1167(2.1 \%)$ & $964(1.7 \%)$ & $213(9.3 \%)$ & $18.3 \%$ & $4.88(4.29-5.55)$ & \\
\hline
\end{tabular}


Table 1. Cont

\begin{tabular}{|c|c|c|c|c|c|c|c|}
\hline Variable & Category & $\begin{array}{c}\text { Total }^{*} \\
n=56,874\end{array}$ & $\begin{array}{c}\text { Non-Hospitalized }^{\circ} \\
n=54,576\end{array}$ & $\begin{array}{c}\text { Hospitalized }^{\circ} \\
\quad n=2298\end{array}$ & $\begin{array}{l}\text { Hospitalized } \\
\text { Row } \%\end{array}$ & $\begin{array}{c}\text { Crude Incidence Rate Ratio } \\
(95 \% \text { Conf. Interv. })\end{array}$ & $p^{\dagger}$ \\
\hline \multirow{2}{*}{ Chronic kidney disease ${ }^{4}$} & No & $54,686(96.2 \%)$ & $52,928(97.0 \%)$ & $1758(76.5 \%)$ & $3.2 \%$ & Reference & \multirow{2}{*}{$<0.0001$} \\
\hline & Yes & $2188(3.8 \%)$ & $1648(3.0 \%)$ & $540(23.5 \%)$ & $24.7 \%$ & $7.68(7.04-8.37)$ & \\
\hline \multirow{2}{*}{ Osteoarthritis } & No & $52,645(92.6 \%)$ & $50,975(93.4 \%)$ & $1670(72.7 \%)$ & $3.2 \%$ & Reference & \multirow{2}{*}{$<0.0001$} \\
\hline & Yes & $4229(7.4 \%)$ & $3601(6.6 \%)$ & $628(27.3 \%)$ & $14.8 \%$ & $4.68(4.29-5.10)$ & \\
\hline \multirow{2}{*}{ Osteoporosis } & No & $55,831(98.2 \%)$ & $53,771(98.5 \%)$ & $2060(89.6 \%)$ & $3.7 \%$ & Reference & \multirow{2}{*}{$<0.0001$} \\
\hline & Yes & $1043(1.8 \%)$ & $805(1.5 \%)$ & $238(10.4 \%)$ & $22.8 \%$ & $6.18(5.49-6.97)$ & \\
\hline \multirow{2}{*}{ Alzheimer/dementia } & No & $56,624(99.6 \%)$ & $54,411(99.7 \%)$ & $2213(93.3 \%)$ & $3.9 \%$ & Reference & \multirow{2}{*}{$<0.0001$} \\
\hline & Yes & $250(0.4 \%)$ & $165(0.3 \%)$ & $85(3.7 \%)$ & $34.0 \%$ & $8.70(7.28-10.39)$ & \\
\hline \multirow{2}{*}{ Epilepsy } & No & $56,465(99.3 \%)$ & $54,216(99.3 \%)$ & $2249(97.9 \%)$ & $4.0 \%$ & Reference & \multirow{2}{*}{$<0.0001$} \\
\hline & Yes & $409(0.7 \%)$ & $360(0.7 \%)$ & $49(2.1 \%)$ & $12.0 \%$ & $3.01(2.31-3.92)$ & \\
\hline \multirow{2}{*}{ Parkinsonism } & No & $56,806(99.9 \%)$ & $54,534(99.9 \%)$ & $2272(98.9 \%)$ & $4.0 \%$ & Reference & \multirow{2}{*}{$<0.0001$} \\
\hline & Yes & $68(0.1 \%)$ & $42(0.1 \%)$ & $26(1.1 \%)$ & $38.2 \%$ & $9.56(7.05-12.97)$ & \\
\hline Stroke (combined) ${ }^{5}$ & Yes & $303(0.5 \%)$ & $225(0.4 \%)$ & $78(3.4 \%)$ & $25.7 \%$ & $6.56(5.39-7.98)$ & $<0.0001$ \\
\hline \multirow{2}{*}{ Stroke (hemorrhagic) } & No & $56,817(99.9 \%)$ & $54,528(99.9 \%)$ & $2289(99.6 \%)$ & $4.0 \%$ & Reference & \multirow{2}{*}{$<0.0001$} \\
\hline & Yes & $57(0.1 \%)$ & $48(0.1 \%)$ & $9(0.4 \%)$ & $15.8 \%$ & $3.92(2.15-7.15)$ & \\
\hline \multirow{2}{*}{ Stroke (ischemic) } & No & $56,705(99.7 \%)$ & $54,453(99.8 \%)$ & $2252(98.0 \%)$ & $4.0 \%$ & Reference & \multirow{2}{*}{$<0.0001$} \\
\hline & Yes & $169(0.3 \%)$ & $123(0.2 \%)$ & $46(2.0 \%)$ & $27.2 \%$ & $6.85(5.34-8.80)$ & \\
\hline \multirow{2}{*}{ Transitory ischemic attack } & No & $56,773(99.8 \%)$ & $54,504(99.9 \%)$ & $2269(98.7 \%)$ & $4.0 \%$ & Reference & \multirow{2}{*}{$<0.0001$} \\
\hline & Yes & $101(0.2 \%)$ & $72(0.1 \%)$ & $29(1.3 \%)$ & $28.7 \%$ & $7.18(5.27-9.79)$ & \\
\hline \multirow{2}{*}{ Asthma } & No & $49,270(86.6 \%)$ & $47,435(86.9 \%)$ & $1835(79.9 \%)$ & $3.7 \%$ & Reference & \multirow{2}{*}{$<0.0001$} \\
\hline & Yes & 7604 (13.4\%) & 7141 (13.1\%) & $463(20.1 \%)$ & $6.1 \%$ & $1.63(1.48-1.81)$ & \\
\hline
\end{tabular}


Table 1. Cont.

\begin{tabular}{|c|c|c|c|c|c|c|c|}
\hline Variable & Category & $\begin{array}{c}\text { Total * } \\
n=56,874\end{array}$ & $\begin{array}{c}\text { Non-Hospitalized }{ }^{\circ} \\
n=54,576\end{array}$ & $\begin{array}{c}\text { Hospitalized }^{\circ} \\
\quad n=2298\end{array}$ & $\begin{array}{l}\text { Hospitalized } \\
\text { Row } \%\end{array}$ & $\begin{array}{l}\text { Crude Incidence Rate Ratio } \\
\text { (95\% Conf. Interv.) }\end{array}$ & $p^{\dagger}$ \\
\hline \multirow{2}{*}{$\begin{array}{l}\text { Chronic obstructive } \\
\text { pulmonary disease }\end{array}$} & No & $55,800(98.1 \%)$ & 53,767 (98.5\%) & $2033(88.5 \%)$ & $3.6 \%$ & Reference & \multirow{2}{*}{$<0.0001$} \\
\hline & Yes & $1074(1.9 \%)$ & $809(1.5 \%)$ & $265(11.5 \%)$ & $24.7 \%$ & $6.77(6.05-7.58)$ & \\
\hline \multirow{2}{*}{ Injection drug use ${ }^{6}$} & No & $54,574(96.0 \%)$ & $52,506(96.2 \%)$ & $2068(90.0 \%)$ & $3.8 \%$ & Reference & \multirow{2}{*}{$<0.0001$} \\
\hline & Yes & $2300(4.0 \%)$ & $2070(3.8 \%)$ & $230(10.0 \%)$ & $10.0 \%$ & $2.64(2.32-3.00)$ & \\
\hline Problematic alcohol use ${ }^{7}$ & No & $54,322(95.5 \%)$ & $52,336(95.9 \%)$ & $1986(86.4 \%)$ & $3.7 \%$ & Reference & $<0.0001$ \\
\hline \multirow{2}{*}{ Cancer $^{8}$} & No & $50,865(89.4 \%)$ & $49,182(90.1 \%)$ & $1683(73.2 \%)$ & $3.3 \%$ & Reference & \multirow{2}{*}{$<0.0001$} \\
\hline & Yes & $6009(10.6 \%)$ & $5394(9.9 \%)$ & $615(26.8 \%)$ & $10.2 \%$ & $3.09(2.83-3.38)$ & \\
\hline \multirow{2}{*}{ Cirrhosis $^{7}$} & No & $56,635(99.6 \%)$ & $54,402(99.7 \%)$ & $2233(97.2 \%)$ & $3.9 \%$ & Reference & \multirow{2}{*}{$<0.0001$} \\
\hline & Yes & $239(0.4 \%)$ & $174(0.3 \%)$ & $65(2.8 \%)$ & $27.2 \%$ & $6.90(5.58-8.52)$ & \\
\hline
\end{tabular}

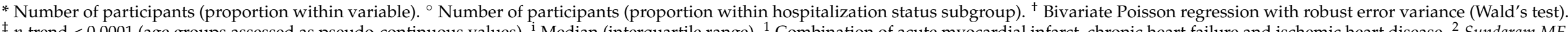

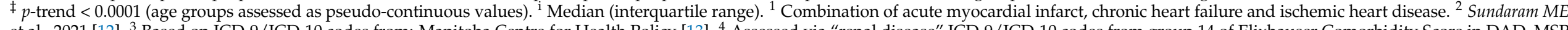

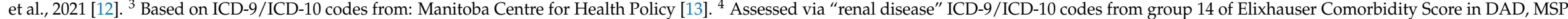

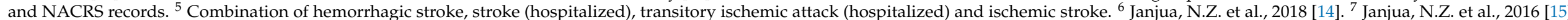

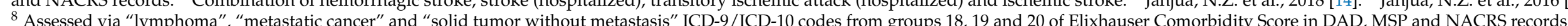


Table 2. Factors associated with hospitalization status in multivariable Poisson regression analysis with robust error variance among confirmed cases, BC COVID-19 Cohort.

\begin{tabular}{|c|c|c|c|}
\hline \multirow{2}{*}{ Variable } & \multirow[t]{2}{*}{ Category } & \multicolumn{2}{|c|}{$\begin{array}{l}26 \text { January } 2020-15 \text { January } 2021 \\
(n=56,874 ; \text { Hospitalized }=2298)\end{array}$} \\
\hline & & $\operatorname{aRR}(95 \% \mathrm{CI}) *$ & $p^{\dagger}$ \\
\hline Sex (vs. female) & Male & 1.27 (1.17-1.37) & $<0.0001$ \\
\hline \multirow{7}{*}{$\begin{array}{c}\text { Age } \ddagger \\
\text { (Reference group: } \\
\text { 20-29 years) }\end{array}$} & $<20$ years & $0.51(0.30-0.85)$ & 0.0103 \\
\hline & 30-39 years & $3.06(2.32-4.03)$ & $<0.0001$ \\
\hline & $40-49$ years & $4.45(3.40-5.82)$ & $<0.0001$ \\
\hline & $50-59$ years & $8.05(6.22-10.41)$ & $<0.0001$ \\
\hline & 60-69 years & 14.98 (11.58-19.37) & $<0.0001$ \\
\hline & $70-79$ years & $28.15(21.64-36.61)$ & $<0.0001$ \\
\hline & $80+$ years & $43.68(33.41-57.10)$ & $<0.0001$ \\
\hline \multicolumn{2}{|c|}{ Asthma } & $1.15(1.04-1.26)$ & 0.0049 \\
\hline \multicolumn{2}{|c|}{ Cancer $^{1}$} & $1.19(1.09-1.29)$ & 0.0001 \\
\hline \multicolumn{2}{|c|}{ Chronic kidney disease $^{2}$} & 1.32 (1.19-1.47) & $<0.0001$ \\
\hline \multirow{2}{*}{$\begin{array}{l}\text { Diabetes (vs. } \\
\text { non-diabetic) }\end{array}$} & Non-insulin & $1.13(1.03-1.25)$ & 0.0112 \\
\hline & Insulin ${ }^{3}$ & $5.05(4.43-5.76)$ & $<0.0001$ \\
\hline \multicolumn{2}{|c|}{ Hypertension } & $1.19(1.08-1.31)$ & 0.0007 \\
\hline \multicolumn{2}{|c|}{ Immunosuppression $^{4}$} & $1.30(1.10-1.54)$ & 0.0019 \\
\hline \multicolumn{2}{|c|}{ Injection drug use ${ }^{5}$} & $2.51(2.14-2.95)$ & $<0.0001$ \\
\hline \multicolumn{2}{|c|}{ Intellectual and developmental disability ${ }^{6}$} & $1.67(1.05-2.66)$ & 0.0307 \\
\hline \multicolumn{2}{|c|}{ Problematic alcohol use ${ }^{7}$} & $1.63(1.43-1.85)$ & $<0.0001$ \\
\hline \multicolumn{2}{|c|}{ Schizophrenia and psychotic disorders } & $1.49(1.23-1.82)$ & $<0.0001$ \\
\hline
\end{tabular}

* Incidence rate ratios adjusted for the variables present in the table. ${ }^{\dagger}$ Wald's test. ${ }^{\ddagger} p$-trend $>0.0001$ (age groups assessed as pseudo-continuous values) ${ }^{1}$ Assessed via "lymphoma", "metastatic cancer" and "solid tumor without metastasis" ICD-9/ICD-10 codes from groups 18, 19 and 20 of Elixhauser Comorbidity Score in DAD, MSP and NACRS records. ${ }^{2}$ Assessed via "renal disease" ICD-9/ICD-10 codes from group 14 of Elixhauser Comorbidity Score in DAD, MSP and NACRS records. ${ }^{3}$ Any type; includes concomitant treatment with antihyperglycemic agents. ${ }^{4}$ Sundaram, M.E. et al., 2021 [12]. ${ }^{5}$ Janjua, N.Z.et al., 2018 [14]. ${ }^{6}$ Based on ICD-9/ICD-10 codes from Manitoba Centre for Health Policy [13]. ${ }^{7}$ Janjua, N.Z. et al., 2016 [15].

Among women of reproductive age (Table 3$)$, pregnancy $(\mathrm{aRR}=3.05 ; 95 \% \mathrm{CI}=1.86-$ $5.07)$, older age $(p$-trend $<0.0001$ across age groups; $\mathrm{RR}=2.32 ; 95 \% \mathrm{CI}=1.54-3.48$ comparing $40-49$ to $20-29$ years-old groups), asthma ( $\mathrm{aRR}=1.80 ; 95 \% \mathrm{CI}=1.29-2.52$ ), diabetes (treated without insulin $\mathrm{aRR}=2.39 ; 95 \% \mathrm{CI}=1.46-3.89$, requiring insulin $\mathrm{aRR}=31.89 ; 95 \% \mathrm{CI}=16.78$ 60.60), hypertension $(\mathrm{aRR}=2.02 ; 95 \% \mathrm{CI}=1.29-3.16)$, IDU $(\mathrm{aRR}=3.97 ; 95 \% \mathrm{CI}=2.44-6.43)$, and problematic alcohol use $(\mathrm{aRR}=3.05 ; 95 \% \mathrm{CI}=1.86-5.02)$, were factors significantly associated with higher risk of hospital admission. 
Sex (vs. female): Male

Age (vs. 20-29 years): <20 years

Age (vs. 20-29 years): 30-39 years

Age (vs. 20-29 years): 40-49 years

Age (vs. 20-29 years): $50-59$ years

Age (vs. 20-29 years): 60-69 years

Age (vs. 20-29 years): $70-79$ years

Age (vs. $20-29$ years): $80+$ years

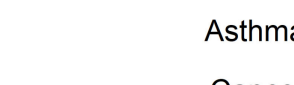

Asthma

Chronic kidney disease

Diabetes (vs. non-diabetic): non-insulin

Diabetes (vs. non-diabetic): insulin

Hypertension

Immunosuppression

Intellectual \& developmental disability

Injection drug use

Problematic alcohol use

Schizophrenia \& psychotic disorders

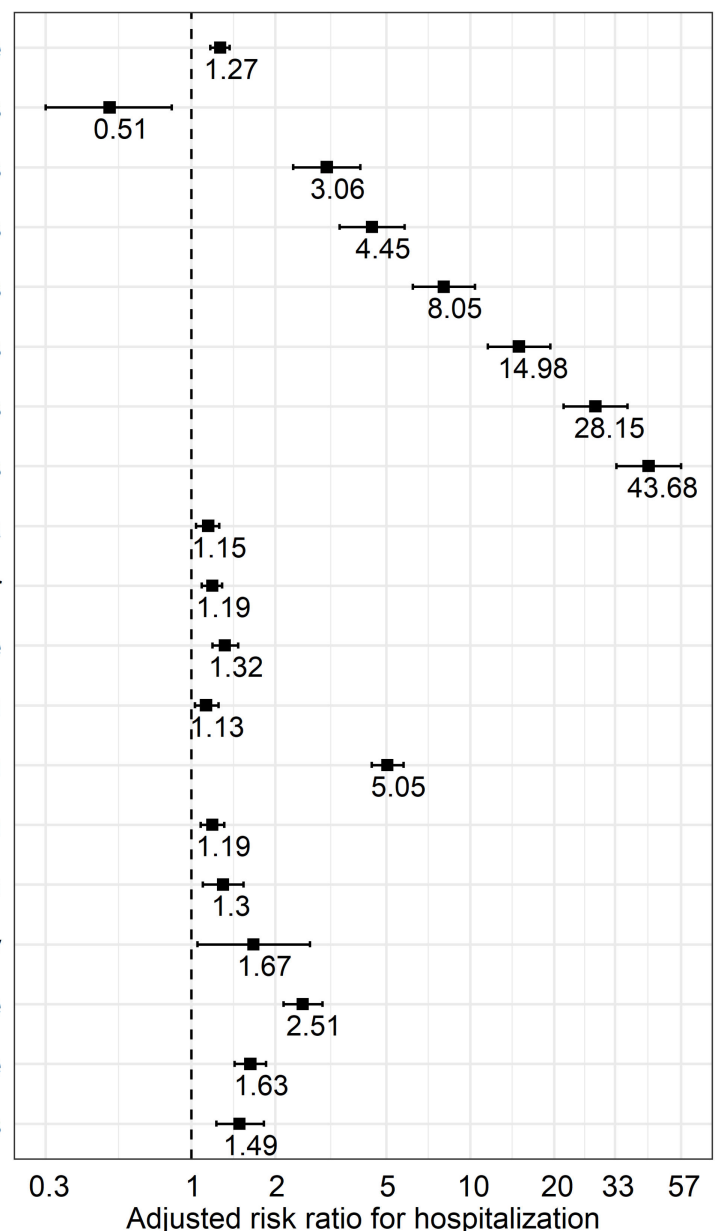

Figure 1. Multivariable model for factors associated with COVID-19-related hospitalization in British Columbia.

Table 3. Factors associated with hospitalization status in multivariable Poisson regression analysis with robust error variance among women of reproductive age (15-49 years-old), BC COVID-19 Cohort $\ddagger$.

\begin{tabular}{|c|c|c|c|}
\hline \multirow{2}{*}{ Variable } & \multirow[t]{2}{*}{ Category } & \multicolumn{2}{|c|}{$\begin{array}{l}26 \text { January } 2020-15 \text { January } 2021 \\
(n=17,036 ; \text { Hospitalized }=191)\end{array}$} \\
\hline & & aRR $(95 \%$ CI $)$ * & $p^{\dagger}$ \\
\hline \multirow{3}{*}{$\begin{array}{c}\text { Age }{ }^{* *} \\
\text { (Reference group: } \\
\text { 20-29 years) }\end{array}$} & $<20$ years & $0.24(0.06-0.95)$ & 0.0424 \\
\hline & 30-39 years & $1.99(1.35-2.94)$ & 0.0005 \\
\hline & 40-49 years & $2.32(1.54-3.48)$ & $<0.0001$ \\
\hline \multicolumn{2}{|c|}{ Asthma } & $1.80(1.29-2.52)$ & 0.0005 \\
\hline \multirow{2}{*}{$\begin{array}{l}\text { Diabetes (vs. } \\
\text { non-diabetic) }\end{array}$} & Non-insulin & $2.39(1.46-3.89)$ & 0.0005 \\
\hline & Insulin ${ }^{1}$ & $31.89(16.78-60.60)$ & $<0.0001$ \\
\hline \multicolumn{2}{|c|}{ Hypertension } & $2.02(1.29-3.16)$ & 0.0020 \\
\hline \multicolumn{2}{|c|}{ Injection drug use ${ }^{2}$} & $3.97(2.44-6.43)$ & $<0.0001$ \\
\hline \multicolumn{2}{|c|}{ Pregnancy } & $2.69(1.42-5.07)$ & 0.0023 \\
\hline \multicolumn{2}{|c|}{ Problematic alcohol use ${ }^{3}$} & $3.05(1.86-5.02)$ & $<0.0001$ \\
\hline
\end{tabular}

$\ddagger 657$ observations with missing pregnancy status were removed from original sample $(n=17,693)$. ${ }^{*}$ Incidence rate ratios adjusted for the variables present in the table. ${ }^{* *} p$-trend $>0.0001$ (age groups assessed as pseudo-continuous values). ${ }^{\dagger}$ Wald's test. ${ }^{1}$ Any type; includes concomitant treatment with antihyperglycemic agents. ${ }^{2}$ Janjua, N.Z. et al., 2018 [14]. ${ }^{3}$ Janjua, N.Z. et al., 2016 [15]. 
In the analysis stratified by age (Table 4), diabetes, and problematic alcohol use were associated with hospitalization across all age groups, with higher risk among younger groups that decreased with older age. Male sex, cancer and hypertension were associated with higher risk of hospital admission among those over 40 years of age. The analysis restricted to hospitalizations lasting more than two days (Supplementary Table S4) did not show different findings compared with the overall analysis.

Table 4. Factors associated with hospitalization status in multivariable Poisson regression analysis with robust error variance among confirmed cases, BC COVID-19 Cohort, stratified by age group.

\begin{tabular}{|c|c|c|c|c|c|c|c|c|c|}
\hline \multirow{3}{*}{\multicolumn{2}{|c|}{ Variable }} & \multicolumn{8}{|c|}{ Age Group } \\
\hline & & \multicolumn{2}{|c|}{$\begin{array}{c}\text { All Age Groups } \\
\quad(n=56,874 ; \\
\text { Hospitalized = 2298) }\end{array}$} & \multicolumn{2}{|c|}{$\begin{array}{l}<40 \text { Years }(n=31,854 \\
\text { Hospitalized }=261)\end{array}$} & \multicolumn{2}{|c|}{$\begin{array}{c}\text { 40-59 Years }(n=16,383 \\
\text { Hospitalized }=578)\end{array}$} & \multicolumn{2}{|c|}{$\begin{array}{r}60+\text { Years }(n=8637 \\
\text { Hospitalized }=1459)\end{array}$} \\
\hline & & $\begin{array}{c}\text { aRR } \\
(95 \% \mathrm{CI}) *\end{array}$ & $p+$ & $\begin{array}{c}\text { aRR } \\
(95 \% \mathrm{CI}) *\end{array}$ & $p^{\dagger}$ & $\begin{array}{c}\text { aRR } \\
(95 \% \text { CI) }\end{array}$ & $p^{+}$ & $\begin{array}{c}\text { aRR } \\
(95 \% \text { CI) }\end{array}$ & $p^{+}$ \\
\hline \multicolumn{2}{|c|}{ Sex (male vs. female) } & $\begin{array}{c}1.27 \\
(1.17-1.37)\end{array}$ & $<0.0001$ & $\begin{array}{c}0.99 \\
(0.77-1.27)\end{array}$ & 0.93 & $\begin{array}{c}1.46 \\
(1.24-1.72) \\
\end{array}$ & $<0.0001$ & $\begin{array}{c}1.26 \\
(1.15-1.38)\end{array}$ & $<0.0001$ \\
\hline \multicolumn{2}{|c|}{ Asthma } & $\begin{array}{c}1.15 \\
(1.04-1.26)\end{array}$ & 0.0049 & $\begin{array}{c}1.10 \\
(0.78-1.54)\end{array}$ & 0.59 & $\begin{array}{c}1.41 \\
(1.16-1.71)\end{array}$ & 0.0005 & $\begin{array}{c}1.10 \\
(0.98-1.23)\end{array}$ & 0.09 \\
\hline \multicolumn{2}{|c|}{ Cancer $^{1}$} & $\begin{array}{c}1.19 \\
(1.09-1.29)\end{array}$ & 0.0001 & $\begin{array}{c}1.47 \\
(0.99-2.17)\end{array}$ & 0.054 & $\begin{array}{c}1.24 \\
(1.00-1.54)\end{array}$ & 0.0459 & $\begin{array}{c}1.31 \\
(1.19-1.44)\end{array}$ & $<0.0001$ \\
\hline \multicolumn{2}{|c|}{ Chronic kidney disease ${ }^{2}$} & $\begin{array}{c}1.32 \\
(1.19-1.47)\end{array}$ & $<0.0001$ & $\begin{array}{c}2.68 \\
(1.22-5.90)\end{array}$ & 0.0141 & $\begin{array}{c}1.32 \\
(0.97-1.80)\end{array}$ & 0.08 & $\begin{array}{c}1.70 \\
(1.53-1.88)\end{array}$ & $<0.0001$ \\
\hline \multirow{2}{*}{$\begin{array}{l}\text { Diabetes (vs. } \\
\text { non-diabetic) }\end{array}$} & Non-insulin & $\begin{array}{c}1.13 \\
(1.03-1.25)\end{array}$ & 0.0112 & $\begin{array}{c}2.03 \\
(0.98-4.17)\end{array}$ & 0.055 & $\begin{array}{c}1.62 \\
(1.31-1.99)\end{array}$ & $<0.0001$ & $\begin{array}{c}1.01 \\
(0.91-1.12)\end{array}$ & 0.87 \\
\hline & Insulin ${ }^{3}$ & $\begin{array}{c}5.05 \\
(4.43-5.76) \\
\end{array}$ & $<0.0001$ & $\begin{array}{c}20.29(4.69- \\
87.90)\end{array}$ & $<0.0001$ & $\begin{array}{c}19.30 \\
(14.66-25.40)\end{array}$ & $<0.0001$ & $\begin{array}{c}3.94 \\
(3.52-4.41) \\
\end{array}$ & $<0.0001$ \\
\hline \multicolumn{2}{|c|}{ Hypertension } & $\begin{array}{c}1.19 \\
(1.08-1.31)\end{array}$ & 0.0007 & $\begin{array}{c}1.87 \\
(0.98-3.57)\end{array}$ & 0.057 & $\begin{array}{c}1.46 \\
(1.21-1.75)\end{array}$ & $<0.0001$ & $\begin{array}{c}1.31 \\
(1.18-1.46)\end{array}$ & $<0.0001$ \\
\hline \multicolumn{2}{|c|}{ Immunosuppression ${ }^{4}$} & $\begin{array}{c}1.30 \\
(1.10-1.54)\end{array}$ & 0.0019 & $\begin{array}{c}2.56 \\
(1.67-3.94)\end{array}$ & $<0.0001$ & $\begin{array}{c}1.30 \\
(0.95-1.76)\end{array}$ & 0.10 & $\begin{array}{c}1.14 \\
(0.93-1.38)\end{array}$ & 0.21 \\
\hline \multicolumn{2}{|c|}{ Injection drug use ${ }^{5}$} & $\begin{array}{c}2.51 \\
(2.14-2.95)\end{array}$ & $<0.0001$ & $\begin{array}{c}2.95 \\
(1.78-4.90)\end{array}$ & $<0.0001$ & $\begin{array}{c}2.55 \\
(1.97-3.30)\end{array}$ & $<0.0001$ & $\begin{array}{c}1.01 \\
(0.76-1.32)\end{array}$ & 0.97 \\
\hline \multicolumn{2}{|c|}{$\begin{array}{l}\text { Intellectual and developmental } \\
\text { disability } 6\end{array}$} & $\begin{array}{c}1.67 \\
(1.05-2.66)\end{array}$ & 0.0307 & $\begin{array}{c}1.17 \\
(0.56-2.42)\end{array}$ & 0.68 & $\begin{array}{c}4.28 \\
(2.29-8.01)\end{array}$ & $<0.0001$ & $\begin{array}{c}1.21 \\
(0.71-2.04)\end{array}$ & 0.49 \\
\hline \multicolumn{2}{|c|}{ Problematic alcohol use ${ }^{7}$} & $\begin{array}{c}1.63 \\
(1.43-1.85)\end{array}$ & $<0.0001$ & $\begin{array}{c}2.84 \\
(1.72-4.72)\end{array}$ & $<0.0001$ & $\begin{array}{c}1.62 \\
(1.26-2.10)\end{array}$ & 0.0002 & $\begin{array}{c}1.53 \\
(1.30-1.79)\end{array}$ & $<0.0001$ \\
\hline \multicolumn{2}{|c|}{$\begin{array}{c}\text { Schizophrenia and psychotic } \\
\text { disorders }\end{array}$} & $\begin{array}{c}1.49 \\
(1.23-1.82)\end{array}$ & $<0.0001$ & $\begin{array}{c}2.33 \\
(1.40-3.87)\end{array}$ & 0.0012 & $\begin{array}{c}1.19 \\
(0.80-1.76)\end{array}$ & 0.38 & $\begin{array}{c}1.56 \\
(1.21-2.00)\end{array}$ & 0.0005 \\
\hline
\end{tabular}

* Incidence rate ratios adjusted for the variables present in the table. ${ }^{\circ}$ Adjusted for also for age (as categorical groups from Table 2 ; not shown). " Wald's test. ${ }^{1}$ Assessed via "lymphoma", "metastatic cancer" and "solid tumor without metastasis" ICD-9/ICD-10 codes from groups 18, 19 and 20 of Elixhauser Comorbidity Score in DAD, MSP and NACRS records. ${ }^{2}$ Assessed via "renal disease" ICD-9/ICD-10 codes from group 14 of Elixhauser Comorbidity Score in DAD, MSP and NACRS records. ${ }^{3}$ Any type; includes concomitant treatment with antihyperglycemic agents. ${ }^{4}$ Sundaram, M.E. et al., 2021 [12]. ${ }^{5}$ Janjua, N.Z. et al., 2018 [14]. ${ }^{6}$ Based on ICD-9/ICD-10 codes from Manitoba Centre for Health Policy [13]. ${ }^{7}$ Janjua, N.Z. et al., 2016 [15].

\section{Discussion}

In this large population-based analysis of all COVID-19 cases in BC, we identified several risk factors for COVID-19 hospitalization. Older age was the strongest predictor of hospital admission, with risk increasing more than 40 fold for the oldest group compared to the reference (20-29 years-old). In addition to well-characterized co-morbidities, we found that injection drug use [5], problematic alcohol use, schizophrenia and psychotic disorders [16] as well as intellectual and developmental disability were independently associated with higher risk of hospitalization. These findings have important implications for vaccination programs. For instance, this evidence was used to identify priority 
groups, informing the COVID-19 vaccination program in BC [17], with the ultimate aim of preventing infection and severe outcomes as well as reducing hospital burden.

Our analysis confirms findings from other studies evaluating risk factors for severe COVID-19 outcomes, although ours is one of the few population-based analyses (i.e., includes all COVID-19 diagnoses in a jurisdiction). Most evaluations have also focused on in-hospital mortality, rather than morbidity. In addition to older age and male sex, a wide range of co-morbidities were associated with a higher risk of hospitalization, reflecting similar findings from other studies [7,18-23]. These comorbidities included asthma, chronic kidney disease, diabetes, cancer, immunosuppression and substance use. Relative associations between most co-morbidities and hospitalization risk were stronger at younger ages, highlighting the overall low absolute risk of hospitalization among younger people without pre-existing comorbidities. However, hospitalization risk increased with older age in the overall population, and the highest absolute risk was observed in people of older ages with co-morbidities. Several biological studies have identified sex and age differences in biological pathways related to SARS-CoV-2 infection and support our findings [24-26].

As in our study, pregnancy has been previously identified as a potential risk factor for ICU admission $[27,28]$ and severe disease [29]. However, most other studies have been limited to pregnant women who were already hospitalized (including for non-COVID-19 reasons such as childbirth) $[27,30]$. This finding could be in part the result of a lower clinical threshold for hospitalization of pregnant patients.

In our study, insulin-dependent diabetes was associated with higher risk of hospitalization, particularly among individuals younger than 40 years. To our knowledge, this is the first report observing this phenomenon, although insulin use and increased risk of COVID-19-related death was described earlier in the literature. Further research is needed to better characterize this finding.

Our study also highlights the intersection between the two ongoing public health emergencies in BC: the COVID-19 and the opioid overdose epidemics. The COVID-19 pandemic has exacerbated the pre-existing opioid epidemic in several ways, including disruption of harm reduction services [31], with BC experiencing a record high number of illicit drug toxicity deaths in 2020 [32]. This is the first study investigating the impact of COVID-19 on people who inject drugs. Our findings suggest that individuals at high risk of overdose, as indicated by IDU, are also at higher risk of COVID-19 hospitalization. IDU was the third strongest predictor of hospitalization in our analysis (following older age and insulin-dependent diabetes). Similarly, problematic alcohol use, schizophrenia and psychotic disorders were also associated with higher risk of hospitalization. These findings may highlight the syndemic of substance use, mental illness and COVID-19. Underlying social conditions (e.g., unstable housing, lower socioeconomic status) and many co-occurring co-morbidities may have exacerbated the effect of COVID-19 infection among these individuals. Prioritization of vaccination for this population group could reduce disparities and decrease risk of hospitalization.

Our analysis had several limitations. We relied on administrative data to identify patient characteristics and co-morbidities; this may have led to some level of misclassification. Similarly, for the same reason, it is not possible to evaluate clinical severity of the event leading to hospital admission. Further, we did not have information on socioeconomic status, race/ethnicity, obesity, and stage of pregnancy (trimester). Moreover, given that the evaluation of the COVID-19 status depends on the BC diagnostic testing guidelines (varying over time to focus on symptom-based assessment since 21 April 2020) [33], selective ascertainment of symptomatic cases is expected, resulting in exclusion of asymptomatic cases [34].

\section{Conclusions}

In conclusion, older age, male sex, pregnancy, and various comorbidities and healthconditions, including substance use, were associated with higher risk of hospital admission 
in this population-based analysis. These findings have informed the COVID-19 vaccination program rollout in $\mathrm{BC}$ and can be useful for informing the prioritization of vaccination in other jurisdictions to prevent infection and severe outcomes [17]. In addition, these findings could also guide healthcare providers in the monitoring of individual patients at higher risk of severe outcomes. Finally, the evidence shows the need for further characterizing syndemics of substance use, mental illness and COVID-19.

Supplementary Materials: The following are available online at https://www.mdpi.com/article/10 .3390/v13112196/s1, Appendix A: BC COVID-19 Cohort, Table S1: Data Sources integrated within the BC COVID-19 Cohort (BCC19C), Appendix B: Obstetric codes (liveborn and stillborn), Appendix C: Definitions for comorbidity variables derived from administrative datasets, Figure S1. Analytic sample flow diagram, Table S2: Factors associated with hospitalization status in multivariable Poisson regression analysis with robust error variance among confirmed cases, BC COVID-19 Cohort, stratified by time period, Table S3: Factors associated with hospitalization status in multivariable Poisson regression analysis with robust error variance among women of reproductive age (15-49 years-old), BC COVID-19 Cohort, stratified by time period, Table S4: Factors associated with hospitalization status in multivariable Poisson regression analysis with robust error variance among confirmed cases, BC COVID-19 Cohort, restricted to hospital admissions lasting more than two days.

Author Contributions: Conceptualization, H.A.V.G. and N.Z.J.; methodology, H.A.V.G., N.Z.J., C.R. and M.O.; software, H.A.V.G.; validation, H.A.V.G. and N.Z.J.; formal analysis, H.A.V.G. and N.Z.J.; investigation, H.A.V.G. and J.W.; resources, N.Z.J., M.K. and N.P.; data curation, N.Z.J., S.D., E.G., J.W., M.C. and M.K.; writing—original draft preparation, H.A.V.G.; writing—review and editing, H.A.V.G., J.W., K.S., M.C., D.R., G.M., S.D., E.G. and N.Z.J.; visualization, H.A.V.G.; supervision, N.Z.J.; project administration, N.Z.J.; funding acquisition, N.Z.J., N.P. and M.K. All authors have read and agreed to the published version of the manuscript.

Funding: This research was funded by the BC Centre for Disease Control and the Canadian Institutes of Health Research [Grant \# VR5-172683 and OV4-170361].

Institutional Review Board Statement: The study was conducted according to the guidelines of the Declaration of Helsinki and approved by the Behavioural Research Ethics Board at the University of British Columbia (approval \# H20-02097).

Informed Consent Statement: This study was performed using data collected as part of public health surveillance or and secondary use of data collected as part of routine healthcare encounters. Data available for analyses was anonymized.

Data Availability Statement: The study is based on data contained in various provincial registries and databases. Access to data could be requested through the BC Centre for Disease Control Institutional Data Access for researchers who meet the criteria for access to confidential data. Requests for the data may be sent to datarequest@bccdc.ca.

Acknowledgments: We acknowledge the assistance of the Provincial Health Services Authority, BC Centre for Disease Control, BC Ministry of Health and Regional Health Authority staff involved in data access, procurement, and management. We thank Naomi Dove and Hasina Samji for their SZP identification algorithm. We gratefully acknowledge the residents of British Columbia whose data are integrated in the British Columbia COVID-19 Cohort (BCC19C).

Conflicts of Interest: M.K. has received grant funding via his institution from Roche Molecular Systems, Boehringer Ingelheim, Merck, Siemens Healthcare Diagnostics and Hologic Inc. All other authors have no potential conflicts of interest to declare. The funders had no role in the design of the study; in the collection, analyses, or interpretation of data; in the writing of the manuscript, or in the decision to publish the results.

Disclaimer: All inferences, opinions, and conclusions drawn in this report are those of the authors, and do not reflect the opinions or policies of the Data Steward(s). 


\section{References}

1. Phua, J.; Weng, L.; Ling, L.; Egi, M.; Lim, C.M.; Divatia, J.V.; Shrestha, B.R.; Arabi, Y.M.; Ng, J.; Gomersall, C.D.; et al. Intensive care management of coronavirus disease 2019 (COVID-19): Challenges and recommendations. Lancet Respir. Med. 2020, 8 , 506-517. [CrossRef]

2. Holman, N.; Knighton, P.; Kar, P.; O’Keefe, J.; Curley, M.; Weaver, A.; Barron, M.; Bakhai, C.; Khunti, K.; Wareham, N.J.; et al. Risk factors for COVID-19-related mortality in people with type 1 and type 2 diabetes in England: A population-based cohort study. Lancet Diabetes Endocrinol. 2020, 8, 823-833. [CrossRef]

3. Carenzo, L.; Costantini, E.; Greco, M.; Barra, F.L.; Rendiniello, V.; Mainetti, M.; Bui, R.; Zanella, A.; Grasselli, G.; Lagioia, M.; et al. Hospital surge capacity in a tertiary emergency referral centre during the COVID-19 outbreak in Italy. Anaesthesia 2020, 75, 928-934. [CrossRef] [PubMed]

4. Himmels, J.P.W.; Borge, T.C.; Brurberg, K.G.; Gravningen, K.M.; Feruglio, S.L.; Berild, J.D. COVID-19 and Risk Factors for Hospital Admission, Severe Disease and Death 3rd Update. Norwegian Institute of Public Health. 15 November 2020. Available online: https:/ / www.fhi.no/en/publ/2020/COVID19-and-risk-factors-for-hospital-admission-severe-disease-and-death-3rd_ update/ (accessed on 18 May 2021).

5. Wang, Q.Q.; Kaelber, D.C.; Xu, R.; Volkow, N.D. COVID-19 risk and outcomes in patients with substance use disorders: Analyses from electronic health records in the United States. Mol. Psychiatry 2021, 26, 30-39. [CrossRef] [PubMed]

6. Baillargeon, J.; Polychronopoulou, E.; Kuo, Y.F.; Raji, M.A. The impact of substance use disorder on COVID-19 outcomes. Psychiatr. Serv. 2021, 72, 578-581. [CrossRef] [PubMed]

7. Ko, J.Y.; Danielson, M.L.; Town, M.; Derado, G.; Greenlund, K.J.; Kirley, P.D.; Alden, N.B.; Yousey-Hindes, K.; Anderson, E.J.; Ryan, P.A.; et al. Risk Factors for Coronavirus Disease 2019 (COVID-19)-Associated Hospitalization: COVID-19-Associated Hospitalization Surveillance Network and Behavioral Risk Factor Surveillance System. Clin. Infect. Dis. 2020, 72, e695-e703. [CrossRef]

8. Owusu, D.; Kim, L.; O’Halloran, A.; Whitaker, M.; Piasecki, A.M.; Reingold, A.; Alden, N.B.; Maslar, A.; Anderson, E.J.; Ryan, P.A.; et al. Characteristics of Adults aged 18-49 Years without Underlying Conditions Hospitalized with Laboratory-Confirmed COVID-19 in the United States, COVID-NET-March-August 2020. Clin. Infect. Dis. 2020, 72, e162-e166. [CrossRef]

9. Sheikh, A.; McMenamin, J.; Taylor, B.; Robertson, C. SARS-CoV-2 Delta VOC in Scotland: Demographics, risk of hospital admission, and vaccine effectiveness. Lancet 2021, 397, 2461-2462. [CrossRef]

10. Zou, G. A Modified Poisson Regression Approach to Prospective Studies with Binary Data. Am. J. Epidemiol. 2004, 159, 702-706. [CrossRef]

11. R Core Team. R: A Language and Environment for Statistical Computing. R Foundation for Statistical Computing, Vienna, Austria. 2020. Available online: https:/ / www.R-project.org/ (accessed on 18 May 2021).

12. Sundaram, M.E.; Calzavara, A.; Mishra, S.; Kustra, R.; Chan, A.K.; Hamilton, M.A.; Djebli, M.; Rosella, L.C.; Watson, T.; Chen, H.; et al. Individual and social determinants of SARS-CoV-2 testing and positivity in Ontario, Canada: A population-wide study. CMAJ 2021, 193, E723-E734. [CrossRef]

13. Manitoba Centre for Health Policy. Concept: Intellectual Disability (ID) (Mental Retardation)/Developmental Disability (DD)/Developmental Disorders. University of Manitoba, 9 July 2020. Available online: http:/ / mchp-appserv.cpe.umanitoba.ca/ viewConcept.php?conceptID=1365 (accessed on 8 February 2021).

14. Janjua, N.Z.; Islam, N.; Kuo, M.; Yu, A.; Wong, S.; Butt, Z.A.; Gilbert, M.; Buxton, J.; Chapinal, N.; Samji, H.; et al. Identifying injection drug use and estimating population size of people who inject drugs using healthcare administrative datasets. Int. J. Drug Policy 2018, 55, 31-39. [CrossRef] [PubMed]

15. Janjua, N.Z.; Kuo, M.; Yu, A.; Alvarez, M.; Wong, S.; Cook, D.; Wong, J.; Grebely, J.; Butt, Z.A.; Samji, H.; et al. The Population Level Cascade of Care for Hepatitis C in British Columbia, Canada: The BC Hepatitis Testers Cohort (BC-HTC). EBioMedicine 2016, 12, 189-195. [CrossRef]

16. Vai, B.; Mazza, M.G.; Delli Colli, C.; Foiselle, M.; Allen, B.; Benedetti, F.; Borsini, A.; Dias, M.C.; Tamouza, R.; Leboyer, M.; et al. Mental disorders and risk of COVID-19-related mortality, hospitalisation, and intensive care unit admission: A systematic review and meta-analysis. Lancet Psychiatry 2021, 8, 797-812. [CrossRef]

17. Government of British Columbia. COVID-19 Immunization Plan. Available online: https://www2.gov.bc.ca/gov/content/ covid-19/vaccine/plan (accessed on 27 June 2021).

18. Atkins, J.L.; Masoli, J.A.H.; Delgado, J.; Pilling, L.C.; Kuo, C.L.; Kuchel, G.A.; Melzer, D. Preexisting Comorbidities Predicting COVID-19 and Mortality in the UK Biobank Community Cohort. J. Gerontol.—Ser. A Biol. Sci. Med. Sci. 2020, 75, 2224-2230. [CrossRef] [PubMed]

19. Galbadage, T.; Peterson, B.M.; Awada, J.; Buck, A.S.; Ramirez, D.A.; Wilson, J.; Gunasekera, R.S. Systematic Review and Meta-Analysis of Sex-Specific COVID-19 Clinical Outcomes. Front. Med. 2020, 7, 348. [CrossRef]

20. O'Brien, J.; Du, K.Y.; Peng, C. Incidence, clinical features, and outcomes of COVID-19 in Canada: Impact of sex and age. J. Ovarian Res. 2020, 13, 137. [CrossRef] [PubMed]

21. Petrilli, C.M.; Jones, S.A.; Yang, J.; Rajagopalan, H.; O’Donnell, L.; Chernyak, Y.; Tobin, K.A.; Cerfolio, R.J.; Francois, F.; Horwitz, L.I. Factors associated with hospital admission and critical illness among 5279 people with coronavirus disease 2019 in New York City: Prospective cohort study. BMJ 2020, 369, m1966. [CrossRef] 
22. Van Gerwen, M.; Alsen, M.; Little, C.; Barlow, J.; Genden, E.; Naymagon, L.; Tremblay, D. Risk factors and outcomes of COVID-19 in New York City; A retrospective cohort study. J. Med. Virol. 2021, 93, 907-915. [CrossRef]

23. Booth, A.; Reed, A.B.; Ponzo, S.; Yassaee, A.; Aral, M.; Plans, D.; Labrique, A.; Mohan, D. Population risk factors for severe disease and mortality in COVID-19: A global systematic review and meta-analysis. PLoS ONE 2021, 16, e0247461. [CrossRef]

24. Haitao, T.; Vermunt, J.V.; Abeykoon, J.; Ghamrawi, R.; Gunaratne, M.; Jayachandran, M.; Narang, K.; Parashuram, S.; Suvakov, S.; Garovic, V.D. COVID-19 and Sex Differences: Mechanisms and Biomarkers. Mayo Clin. Proc. 2020, 95, 2189-2203. [CrossRef]

25. Takahashi, T.; Ellingson, M.K.; Wong, P.; Israelow, B.; Lucas, C.; Klein, J.; Silva, J.; Mao, T.; Oh, J.E.; Tokuyama, M.; et al. Sex differences in immune responses that underlie COVID-19 disease outcomes. Nature 2020, 588, 315-320. [CrossRef] [PubMed]

26. Klein, S.L.; Pekosz, A.; Park, H.S.; Ursin, R.L.; Shapiro, J.R.; Benner, S.E.; Littlefield, K.; Kumar, S.; Naik, H.M.; Betenbaugh, M.J.; et al. Sex, age, and hospitalization drive antibody responses in a COVID-19 convalescent plasma donor population. J. Clin. Investig. 2020, 130, 6141-6150. [CrossRef]

27. Zambrano, L.D.; Ellington, S.; Strid, P.; Galang, R.R.; Oduyebo, T.; Tong, V.T.; Woodworth, K.R.; Nahabedian, J.F., III; AzzizBaumgartner, E.; Gilboa, M.; et al. Update: Characteristics of Symptomatic Women of Reproductive Age with LaboratoryConfirmed SARS-CoV-2 Infection by Pregnancy Status-United States, January 22-October 3, 2020. Morb. Mortal. Wkly. Rep. 2020, 69, 1641-1647. [CrossRef] [PubMed]

28. Badr, D.A.; Mattern, J.; Carlin, A.; Cordier, A.G.; Maillart, E.; El Hachem, L.; El Kenz, H.; Andronikof, M.; De Bels, D.; Damoisel, C.; et al. Are clinical outcomes worse for pregnant women at $\geq 20$ weeks' gestation infected with coronavirus disease 2019? A multicenter case-control study with propensity score matching. Am. J. Obstet. Gynecol. 2020, 223, 764-768. [CrossRef] [PubMed]

29. Oakes, M.C.; Kernberg, A.S.; Carter, E.B.; Foeller, M.E.; Palanisamy, A.; Raghuraman, N.; Kelly, J.C. Pregnancy as a risk factor for severe coronavirus 2019 (COVID-19) disease using standardized clinical criteria. Am. J. Obstet. Gynecol. MFM 2021, $3,100319$. [CrossRef] [PubMed]

30. Allotey, J.; Stallings, E.; Bonet, M.; Yap, M.; Chatterjee, S.; Kew, T.; Debenham, L.; Llavall, A.C.; Dixit, A.; Zhou, D.; et al. Clinical manifestations, risk factors, and maternal and perinatal outcomes of coronavirus disease 2019 in pregnancy: Living systematic review and meta-analysis. BMJ 2020, 370, 3320. [CrossRef]

31. BC Centre for Disease Control. Overdose Response Indicators. Available online: http://www.bccdc.ca/health-professionals/ data-reports / overdose-response-indicators (accessed on 6 July 2021).

32. British Columbia Coroner's Service. Llicit Drug Toxicity Deaths in BC: January 1, 2011-March 31, 2021. Minister of Public Safety and Solicitor General, Burnaby, BC. 29 April 2021. Available online: https://www2.gov.bc.ca/assets/gov/birth-adoption-deathmarriage-and-divorce/deaths/coroners-service/statistical/illicit-drug.pdf (accessed on 6 July 2021).

33. BC Centre for Disease Control. Phases of COVID-19 Testing in BC. 2021. Available online: http://www.bccdc.ca/health-info/ diseases-conditions / covid-19/testing/phases-of-covid-19-testing-in-bc (accessed on 6 July 2021).

34. Yanes-Lane, M.; Winters, N.; Fregonese, F.; Bastos, M.; Perlman-Arrow, S.; Campbell, J.R.; Menzies, D. Proportion of asymptomatic infection among COVID-19 positive persons and their transmission potential: A systematic review and meta-analysis. PLoS ONE 2020, 15, e0241536. [CrossRef] 